\title{
Spotlight on Melphalan Flufenamide: An Up-and- Coming Therapy for the Treatment of Myeloma
}

\author{
Fortunato Morabito, , ${ }^{1}$ Giovanni \\ Tripepi, ${ }^{3}$ Enrica Antonia \\ Martino, ${ }^{4}$ Ernesto Vigna, ${ }^{4}$ \\ Francesco Mendicino, (D) ${ }^{4}$ Lucio \\ Morabito, ${ }^{5}$ Katia Todoerti, ${ }^{6}$ \\ Hamdi Al-Janazreh, ${ }^{2}$ Graziella \\ D’Arrigo, ${ }^{3}$ Filippo Antonio \\ Canale, ${ }^{7}$ Giovanna Cutrona, ${ }^{8}$ \\ Antonino Neri, ${ }^{6,8}$ Massimo \\ Martino, (iD) 9 Massimo Gentile (iD) 4 \\ 'Biotechnology Research Unit, AO of \\ Cosenza, Cosenza, Italy; ${ }^{2}$ Hematology \\ and Bone Marrow Transplant Unit, \\ Hemato-Oncology Department, Augusta \\ Victoria Hospital, East Jerusalem, Israel; \\ ${ }^{3}$ HCNR-IBIM, Clinical Epidemiology and \\ Physiopathology of Renal Diseases and \\ Hypertension of Reggio Calabria, Reggio, \\ Calabria, Italy; ${ }^{4} \mathrm{Hematology}$ Unit, AO of \\ Cosenza, Cosenza, Italy; ${ }^{5}$ Humanitas \\ Clinical and Research Center-IRCCS, \\ Rozzano, Milan, Italy; ${ }^{6} \mathrm{Hematology,}$ \\ Fondazione Cà Granda IRCCS Policlinico, \\ Milan, Italy; ${ }^{7}$ Stem Cell Transplant \\ Program, Clinical Section, Department of \\ Hemato-Oncology and Radiotherapy, \\ Grande Ospedale Metropolitano \\ "Bianchi-Melacrino-Morelli", Reggio, \\ Calabria, Italy; ${ }^{8}$ IRCCS Ospedale \\ Policlinico San Martino, Genoa, Italy; \\ ${ }^{9}$ Department of Oncology and Hemato- \\ oncology, University of Milan, Milan, Italy
}

Correspondence: Fortunato Morabito Biotechnology Research Unit, AO of Cosenza, Contrada San Nicola, Cosenza, Italy, Cosenza, 87100, Italy

Tel +39-0984-015863

Fax +39-0984-68I329

Email f.morabito53@gmail.com

Massimo Gentile

Hematology Unit, AO of Cosenza, Italy, viale della Repubblica snc, Cosenza, 87100 , Italy

Tel +39-0984-68I 329

Fax +39-0984-681329

Email massim.gentile@tiscali.it
Abstract: Despite recent therapeutic advances, multiple myeloma (MM) patients experience relapses as they become resistant to various classes and combinations of treatment. Melphalan (L-PAM) is an ageless drug. However, its use in the autologous stem cell transplantation (ASCT) setting and the innovative quadruplet regimen as well as daratumumab, bortezomib, and prednisone make this old drug current yet. Melflufen is a peptideconjugated alkylator belonging to a novel class of compounds, representing an overcoming of L-PAM in terms of mechanism of action and effectiveness. The improved melflufen cytotoxicity is related to aminopeptidase activity, notably present in normal and neoplastic cells and remarkably heavily overexpressed in MM cells. Upon entering a cell, melflufen is cleaved by aminopeptidases, ultimately releasing the L-PAM payload and eliciting further the inflow and cleavage of the conjugated peptide. This virtuous loop persists until all extracellular melflufen has been utilized. The aminopeptidase-driven accumulation results in a 50-fold increase in L-PAM cell enrichment as compared with free alkylator. This condition produces selective cytotoxicity, increased on-target cell potency, and decreased offtarget cell toxicity, ultimately overcoming resistance pathways triggered by previous treatments, including alkylators. Due to its distinct mechanism of action, melflufen plus dexamethasone as a doublet, and in combination with other novel drugs, has the potential to be beneficial for a broad range of patients with relapsed/refractory (RR) MM in third- or even in second-line therapy. The safety profile of melflufen has been consistent across studies, and no new safety concerns have been identified when melflufen was administered in doublet and triplet combinations. Based on growing clinical evidence, melflufen could be not only a good addition in the fight against RRMM but also a drug with a very favorable tolerability profile. Keywords: multiple myeloma, relapsed resistant, melphalan flufenamide, melflufen, therapy, melphalan

\section{Introduction}

The treatment of multiple myeloma $(\mathrm{MM})$ is presently experiencing an improved therapeutic algorithm evolution. Numerous new drugs are available and wellintegrated into modern MM therapy. ${ }^{1-16}$ In this respect, the first and subsequent treatment lines are enriched with a suitable combination of these novel agents in doublets, triplets, and quadruplets, with proper class shift scheduling. ${ }^{1-16}$ Novel chimeric antigen receptor (CAR) T cell-based therapies, ${ }^{17}$ along with anti-B-cell maturation antigen (BCMA) antibody-drug conjugates, bispecific antibodies, and bispecific T cell engagers (BiTEs), ${ }^{18}$ produce renewed expectation for MM patients. Novel antibody constructs, such as belantamab mafodotin, ${ }^{19}$ can be considered after repeated relapses. Overall, this incredible therapeutic armamentarium allows an 
optimal therapeutic strategy for naïve and relapsed resistant (RR) MM and physicians' challenges.

The inclusion of these novel therapies into the autologous stem-cell transplantation (ASCT) paradigm as consolidation and comprising induction and maintenance therapies has further improved clinical outcomes. ${ }^{20-24}$ This model represents a mandatory choice for young patients and eligible elderly fit MM patients. ${ }^{20}$

Moreover, some strategies, ie, continuous versus temporally defined treatments, contributed to further improving the natural history of MM patients. Nevertheless, it remains reasonably tricky the inadequacy of trials focusing on crucial questions, such as the delineation of ideal treatment sequencing approaches in MM patients' homogeneous cohorts.

Melphalan (L-PAM) was developed more than a halfcentury ago, ${ }^{25}$ and extensive medical know-how has been gained over time. ${ }^{26,27}$ Among the different agents, L-PAM remains the MM treatment 'prototype, with excellent results in the disease's palliation but without any impact on the disease progression. ${ }^{28}$ Although L-PAM has currently been switched to more novel drugs, its more recent use in the quadruplet regimen with daratumumab plus bortezomib and prednisone makes this drug current yet. ${ }^{29}$ More importantly, the disease control maintenance over time has undoubtedly been improved utilizing the therapeutic combinations, including L-PAM. ${ }^{30}$ Finally, the L-PAM intravenous formulation used at a high dose was subsequently exploited since it would increase the neoplastic cell death occurrence and affect tumor drug resistance. ${ }^{31}$ However, while some achievements, ie, deep responses, are debating whether ASCT should be considered mandatory for eligible newly diagnosed patients, ${ }^{32}$ high-dose L-PAM remains the standard conditioning regimen in ASCT for MM eligible patients. ${ }^{20}$

The pharmacologic selection of alkylating oligopeptides recognized melphalan flufenamide, as a new, more potent anti-tumor drug than L-PAM, although with an equal alkylating aptitude. ${ }^{33-35}$

Melflufen, a first-in-class peptide-drug conjugate (PDC), enters neoplastic cells effortlessly due to lipophilicity and targets aminopeptidases within the cell, ultimately explaining its higher tumor cell, ${ }^{36}$ thus offering a superior cost-benefit ratio than that attained with L-PAM. This assumption would well-fit the therapeutic need of MM patients who have prior exposure/resistance to L-PAM ${ }^{37}$ or a different class of new drugs.
This review aims to illustrate the chemical, the pharmacokinetics, the mechanism of action, the preclinical and the clinical developments of melflufen up to the Food and Drug Administration (FDA) approval on February 26, 2021, in combination with dexamethasone for adult patients with RRMM. ${ }^{38}$

\section{Historical Notes}

Italian researchers synthesized m-L-sarcolysins-based small peptides more than 60 years ago, and a combination of six of them, denominated Peptichemio, entered clinical trials and promoted. ${ }^{39}$ Subsequently, studying Peptichemio individual oligopeptides, attention has been focused on the tripeptide L-prolyl-mL-sarcolysyl-p -L-fluorophenylalanine ethyl ester (P2), which exhibited a more remarkable antitumor activity than the other five components, also in low proliferating index neoplastic cells, ${ }^{40}$ and even more potent compared with L-PAM. ${ }^{41}$ Finally, a different alkylating dipeptide J1 (L-melphalanyl-p-L-fluorophenylalanine ethyl ester, or melflufen), having an intermediate assembly between L-PAM and P2 compounds structurally, demonstrated superior cytotoxic metabolic and apoptosis activities as well as improved delivering properties compared with the single compounds. ${ }^{34}$

\section{Pharmacology and Pharmacokinetics}

Melflufen, previously named J1, is chemically the ethyl ester of a dipeptide consisting of the old alkylator L-PAM and para-fluoro-L-phenylalanine (Figure 1). By adding the alkyl unit to the DNA guanine base, L-PAM induces an abnormal link amongst DNA components, leading to the breakage of DNA and, ultimately, its synthesis inhibition. ${ }^{42}$ Regrettably, L-PAM cannot enter cell membranes effortlessly due to its hydrophilic nature, representing a limitation for fully exerting its antineoplastic potential. Conversely, melflufen, due to its high lipophilicity, penetrates cancer cells quickly, undergoing hydrolysis by aminopeptidases. ${ }^{36}$ Interestingly, CD13 is commonly expressed in cancer cells, ${ }^{43}$ and it exerts a central function in affecting tumor cell development. ${ }^{44}$ The significance of CD13 aminopeptidase activity leading to improved melflufen cytotoxic activity was described in numerous cell types, ${ }^{36}$ including multiple myeloma. ${ }^{45}$ Although CD13 aminopeptidase was consistently validated as a melflufen substrate, the overall evidence of its function in the 
<smiles>CCOC(=O)[C@H](Cc1ccc(F)cc1)NC(=O)[C@H](N)Cc1ccc(N(CCCl)CCCl)cc1</smiles><smiles>[R]OC(=O)[C@H](N)Cc1ccc(F)cc1</smiles>

Melphalan

p-Fluorophenylalanine

Figure I Chemical structure and metabolism of melflufen (melphalan flufenamide) hydrochloride, a dipeptide prodrug of melphalan. Melflufen is metabolized into melphalan and p-Fluorophenylalanine by aminopeptidases (AP) inside myeloma cells. Melflufen utilizes not only intracellular aminopeptidases (AP) but also esterases (ES) to release a melphalan cytotoxic payload in neoplastic myeloma cells.

melflufen activity in MM is limited. It should be emphasized that a detailed study on aminopeptidase gene expression in myeloma revealed that some aminopeptidases were highly expressed in $\mathrm{MM} .{ }^{46}$ Moreover, the high expression of some of these aminopeptidase genes, ie, X-prolyl aminopeptidase 1 (XPNPEP1), Arginyl aminopeptidase (RNPEP), Dipeptidyl peptidase 3 (DPP3), and Bleomycin hydrolase (BLMH), was significantly associated with shorter overall survival. More importantly, hydrolysis analysis demonstrated that melflufen is a substrate of Leucine aminopeptidase 3 (LAP3), leukotriene A4 hydrolase (LTA4H), arginyl aminopeptidase (RNPEP), other than aminopeptidase N (ANPEP alias CD13), suggesting that
CD13 is not the unique melflufen target in MM. Finally, the study of esterase expression in $\mathrm{MM}$ is becoming of increasing interest due to the development of melflufen, which utilizes not only intracellular aminopeptidases but also and esterases to release a L-PAM cytotoxic payload in neoplastic MM cells. ${ }^{37,45,47}$

Thus, because of the fast melflufen transport rate into cells and, conversely, the gradual exit of L-PAM-free outside cells, a high intracellular-free L-PAM concentration is achieved due to the intracellular cleavage. In other words, treatment with melflufen efficiently results in the intracellular trapping of L-PAM. Consequently, the melflufen cytotoxic IC50-value is drastically lower (from 10 to 600- 
fold reduced) than L-PAM. ${ }^{33,34,42}$ Furthermore, the in vitro study of melflufen as well as of other melphalancontaining dipeptide derivatives showed that factors (ie, amino acid composition and sequence, and modifications of the $\mathrm{C}$ - and $\mathrm{N}$-termini of the dipeptide derivatives) seemed to influence the anti-tumor pharmacologic weight strongly, and at a small magnitude, the peptides' lipophilicity, anyway superior to L-PAM. Overall, these data indicated that both the chemical reactivity of melflufen and its active biological interaction should be considered to explain the ultimate antineoplastic result. ${ }^{48}$ Thus, differently from earlier therapies aiming to inhibit aminopeptidase, ${ }^{49}$ melflufen exploits increased aminopeptidase activity in MM cells to selectively direct the alkylator into tumor cells. ${ }^{33}$ Accordingly, peptidase inhibition experiments ${ }^{45}$ showed that the aminopeptidase inhibitor bestatin could block the prompt intracellular release of free L-PAM following melflufen exposure, dropping its accumulation and the consequent cellular toxicity. ${ }^{33}$ Remarkable, melflufen action is gained in half-hour of in vitro exposure, ${ }^{33}$ thus quite independently on drug exposure, which is clinically essential considering the melflufen short half-life in humans. ${ }^{35}$

Pharmacokinetics assessment data were available for 29 patients undergoing 50 cycles of melflufen at a dose ranging from $25 \mathrm{mg}$ to $130 \mathrm{mg}$ in a Phase I-II study on cancer patients. ${ }^{50}$ Since melflufen is eliminated from peripheral blood quickly after the intravenous administration, any calculation on the elimination phase was prevented. While melflufen peak concentration was frequently detected just before the end of the drug infusion, Tmax for L-PAM essentially happened 5-15 min after melflufen infusion end. Thus, L-PAM's delayed peak plasma concentrations were well-matched, assuming its massive formation from melflufen, external of the plasma compartment, following L-PAM dissemination back to blood plasma. A fast transformation of melflufen to melphalan was demonstrated by Cmax and AUC over the infusion time $0-0.5 \mathrm{~h}$, which was significantly greater for melphalan. ${ }^{50}$ After the end of melflufen infusion, melphalan plasma concentrations continue to increase for up to 10 minutes.

\section{Preclinical Studies}

Melflufen activity was tested against numerous tumorderived cell lines with fluctuating capability, ranging from drug amount measurable in $\mathrm{nM}$ for small-cell lung cancer compared with the $\mu \mathrm{M}$ for the non-small-cell lung cancer cell line,${ }^{51}$ with an overall average of half-maximal inhibitory concentration (IC50) of $0.41 \mathrm{uM}$ for both solid and hematological cancer cells. ${ }^{35}$ Nevertheless, these data demonstrated a significantly reduced average of IC50 of melflufen compared to L-PAM (18 uM). ${ }^{35}$ More convincing results were provided by the in vitro study of drugresistant neoplastic cell lines showing roughly 300-fold increased effectiveness of melflufen than L-PAM. ${ }^{52}$

In vitro studies on primary neoplastic cells showed breast cancer, ovarian cancer, and non-small cell lung carcinoma samples to be more prone to the melflufen cytotoxic effect than renal and colon cancers. ${ }^{41}$ Again, the overall average L-PAM IC-50 was significantly higher, ranging from a 75 -fold increase for primary cells derived from ovarian carcinoma to a 700 -fold increase for very aggressive breast tumor cells. ${ }^{41}$ Another set of experiments showed a clear correlation between melflufen's cytotoxic effect and aminopeptidase activity levels in osteosarcoma cells. ${ }^{53}$ Notably, high-CD13 expression was linked to poor survival in osteosarcoma patients. ${ }^{54}$ Again, the inhibition of this cytotoxic effect by bestatin, a general aminopeptidase inhibitor, indicates the central role of aminopeptidase activity is essential for melflufen's antiosteosarcoma effect. ${ }^{53}$ Importantly, melflufen superior cytotoxic activity was also proved in methotrexate- or etoposide-resistant osteosarcoma cells, substantiating evidence of its potential use in removing chemoresistant neoplasia. Notably, the pre-treatment of osteosarcoma cells with doxorubicin, which is able to up-regulate aminopeptidase expression, synergy with melflufen cytotoxic activity, supporting the rationale for a doxorubicinmelflufen combined chemotherapy. Overall, these experiments provided a strategy for targeting even osteosarcoma cells with low CD13 expression, which is expected to be less sensitive to melflufen as a single agent. ${ }^{52}$

Considering the unquestionable role of L-PAM in the treatment of $\mathrm{MM},{ }^{55}$ the studies understandably shifted to myeloma cells. The in vitro analyses were pitted on three different points. First, activity of aminopeptidases was established in MM cell lines as well as the consequent aminopeptidase-dependent melflufen cleavage. ${ }^{44}$ Second, it was demonstrated that the L-PAM-resistant subclone of a myeloma cell-line RPMI-8226 and a doxorubicinresistant cell line were respectively nearby 10 - and 2.5-fold more sensitive to melflufen due to a predictable improved accumulation and exposure to the L-PAM. ${ }^{40}$ The ways melflufen overcome the resistant mechanisms to L-PAM are not fully elucidated, depending on their 
reduced DNA damage, the increased glutathione$\mathrm{S}$-transferase activity, and the cell-adhesion mediated drug resistance. ${ }^{56-59}$ Notably, differently from melflufen, L-PAM positively modulates the DNA healing upregulating $\mathrm{Ku} 80$, which in turn repairs DNA doublestrand breaks in MM cells. ${ }^{60}$ Third, melflufen inhibits myeloma cell migration induced by vascular endothelial growth factor. ${ }^{44}$ Finally, experiments on the in vivo efficacy of melflufen using a human plasmacytoma MM.1S xenograft mouse model showed significant inhibition of myeloma tumor growth, prolonging mouse survival consequently. $^{44}$

These preclinical in vitro and in vivo reports suggest that melflufen can induce faster, stronger, and permanent DNA damage, thus overcoming L-PAM resistance in MM cells. Together with the results mentioned above, the finding that melflufen can generate synergism with the commonly used anti-myeloma drugs (ie, lenalidomide, bortezomib, and dexamethasone $)^{44}$ have paved the way for clinical trials in humans.

\section{Clinical Studies}

\section{First-in-Human Phase I-2 Trial}

A prospective, first-in-human, dose-finding phase I/IIa study was designed in patients with advanced and progressive neoplasia, mainly breast cancer, ovarian cancer, lymphoma, and lung cancer, deprived of any standard treatment choices. ${ }^{50}$ Melflufen was administered in 45 patients as a $30 \mathrm{~min}$ intravenous infusion once every three weeks for up to six cycles, to determine, in Phase I part, the recommended Phase II dose of melflufen and, after its achievement, the goals were to establish the melflufen safety profile, assess the pharmacokinetic profile, and investigate any evidence of tumor response. No doselimiting toxicities were observed at 25 and $50 \mathrm{mg}$, while at higher doses, reversible neutropenia and thrombocytopenia were the main dose-limiting toxicities, remarkably in heavily treated patients. Therefore, the final recommended phase II dose was fixed at $50 \mathrm{mg}$. A partial response was documented in one ovarian cancer case, while 18 patients achieved stable disease. Thus, the authors concluded that melflufen could safely be administered to cancer patients at a dose of $50 \mathrm{mg}$ every three weeks. The toxicity profile was similar to alkylating agents.

\section{Clinical Trials in Multiple Myeloma}

Table 1 shows the main published trials involving RRMM patients treated with melflufen.

\section{The O-I2-MI Trial}

The O-12-M1 (NCT01897714) was an open-label, multicentre, international phase I/II study designed to establish

Table I The Main Published Trials Involving RRMM Patients Treated with Melflufen

\begin{tabular}{|l|l|l|l|l|l|l|l|}
\hline Trial & Schedule & $\begin{array}{l}\text { No of } \\
\text { Patients }\end{array}$ & $\begin{array}{l}\text { Median } \\
\text { Prior } \\
\text { Therapies }\end{array}$ & $\begin{array}{l}\text { Median } \\
\text { Follow- } \\
\text { Up } \\
\text { (Months) }\end{array}$ & $\begin{array}{l}\text { ORR } \\
\text { (\%) }\end{array}$ & $\begin{array}{l}\text { Median } \\
\text { PFS } \\
\text { (Months) }\end{array}$ & Safety Profile \\
\hline $\begin{array}{l}\text { O-I2-MI } \\
\text { (Phase I/II) } \\
(6 I, 62)\end{array}$ & $\begin{array}{l}\text { Melflufen + } \\
\text { dexamethasone }\end{array}$ & $\begin{array}{l}45 \text { (Phase } \\
\text { II; evaluable } \\
\text { for } \\
\text { response) }\end{array}$ & $4(2-14)$ & 27.9 & 31 & 5.7 & $\begin{array}{l}\text { Grade 3/4 TRAEs: thrombocytopenia (58\%), } \\
\text { neutropenia (58\%), anemia (42\%); serious } \\
\text { TRAEs: 27\% (pneumonia 9\%) }\end{array}$ \\
\hline $\begin{array}{l}\text { HORIZON } \\
\text { (Phase II) } \\
(63)\end{array}$ & $\begin{array}{l}\text { Melflufen + } \\
\text { dexamethasone }\end{array}$ & 157 & $5(2-12)$ & 14 & 29 & 4.2 & $\begin{array}{l}\text { Grade 3/4 TRAEs: neutropenia (79\%), } \\
\text { thrombocytopenia (76\%), anemia (43\%); } \\
\text { serious TRAEs: 49\% (pneumonia 9\%) }\end{array}$ \\
\hline $\begin{array}{l}\text { ANCHOR } \\
\text { (Phase I/II) } \\
(64)\end{array}$ & $\begin{array}{l}\text { Melflufen + } \\
\text { dexamethasone } \\
+ \text { daratumumab }\end{array}$ & 33 & $2(1-4)$ & $I I .9$ & 70 & 11.5 & $\begin{array}{l}\text { No DLTs grade 3/4 TRAEs: neutropenia } \\
\text { (58\%), thrombocytopenia (55\%), anemia } \\
\text { (24\%); serious TRAEs: 36\% (influenza 9\%) }\end{array}$ \\
\cline { 2 - 7 } & $\begin{array}{l}\text { Melflufen + } \\
\text { dexamethasone } \\
+ \text { bortezomib }\end{array}$ & 10 & $2.5(I-4)$ & NR & 60 & NR & $\begin{array}{l}\text { No DLTs grade 3/4 TRAEs: } \\
\text { thrombocytopenia (80\%), neutropenia (60\%), } \\
\text { anemia (40\%); serious TRAEs: 60\% } \\
\text { (pneumonia 20\%) }\end{array}$ \\
\hline
\end{tabular}

Abbreviations: No, number; ORR, overall response rate; PFS, progression-free survival; NR, not reported; DLT, dose-limiting toxicity; TRAE, treatment-related adverse event. 
the dose and the schedule of melflufen in combination with dexamethasone and of evaluating the response in RRMM patients who were refractory to the last line of therapy. Seventy-five heavily pretreated MM patients were enrolled in this trial. ${ }^{61}$

In Phase 1 of the study, melflufen was administered intravenously for 30 minutes at four dose levels $(15,25$, 40 , and $55 \mathrm{mg}$ ) combined with oral dexamethasone $40 \mathrm{mg}$ weekly 21-day cycles. Since 4 of 6 patients receiving $55 \mathrm{mg}$ dose experienced Grade 4 dose-limiting hematologic toxicities, the recommended dose for expansion was $40 \mathrm{mg}$. In Phase 2, 58 patients were enrolled; 45 patients received $40 \mathrm{mg}$ melflufen on day 1 of each cycle plus $40 \mathrm{mg}$ once-week dexamethasone in 21-day cycles, and 13 patients received single-agent $40 \mathrm{mg}$ melflufen on day 1 of each cycle. After the enrollment of the first 28 (62\%) patients in the combination cohort, the Data Safety Monitoring Committee amended the protocol increasing the cycle length to 28 days to allow the hematologic recovery between cycles. Thus, the following 17 (38\%) patients received therapy with 28 -day cycles. ${ }^{61}$

The ORR [ $\geq$ partial response (PR)] was $31 \%(14 / 45$ cases), with a median duration of response of 8.4 months. The ORR was $41 \%$ among patients who received $>2$ doses (14/34 cases) of study treatment. Patients who received melflufen alone achieved an ORR of $8 \%$. At a median follow-up of 27.9 months, the median duration of response (DOR) was 8.4 months, the median PFS was 5.7 months, and the median overall survival (OS) was 20.7 months. ${ }^{61}$ In a subsequent analysis, with a median follow-up of 46 months, the median OS was also 20.7 months, and the median time-to-next treatment 7.9 months. ${ }^{62}$

The most common adverse events (AEs) were thrombocytopenia $(73 \%)$, neutropenia $(69 \%)$, anemia $(64 \%)$, pyrexia (40\%), and asthenia (31\%).

Serious AEs (SAEs) occurred in 38\% of patients; the most common SAE was pneumonia (11\%). The most common melflufen-related grade $3 / 4$ AEs were most commonly reversible thrombocytopenia (58\%) and neutropenia (58\%). Thus, the combination of melflufen and dexamethasone was generally manageable in this setting of heavily pretreated MM. ${ }^{61}$

\section{The HORIZON Trial (OP-I06)}

HORIZON (OP-106) is a pivotal, single-arm, multicenter, Phase II study designed to evaluate the efficacy and safety of melflufen and dexamethasone in heavily pretreated RRMM patients refractory to pomalidomide and/or an
anti-CD38 monoclonal antibody. ${ }^{63}$ Patients received melflufen $40 \mathrm{mg}$ intravenously on day 1 of each 28-day cycle plus oral dexamethasone ( $40 \mathrm{mg}$ or $20 \mathrm{mg}$ in patients older than 75 years, weakly). One hundred and fifty-seven patients were enrolled. The median age was 65 years; the median of prior lines of therapy was 5. Of 157 patients, $119(75.8 \%)$ cases were triple-class-refractory, and 97\% were refractory to treatment in the last line. The ORR was $29 \%$, with $26 \%$ in the triple-class-refractory setting. At a median follow-up of 14 months, the median duration of response, median progression-free survival, and median overall survival were 5.5 months, 4.2 months, and 11.6 months, respectively. The most common Grade 3/4 treatment-emergent AE was hematologic events: neutropenia (79\%), thrombocytopenia (76\%), and anemia (43\%), while the most common grade 3/4 nonhematologic AE was pneumonia $(10 \%){ }^{63}$

This trial, as well as the O-12-M1, confirmed the clinical efficacy and manageable safety profile of melflufen and dexamethasone combination in heavily pretreated RRMM patients, including those with triple-classrefractory. ${ }^{63}$

\section{The ANCHOR Trial (OP-I04)}

Based on the assumption that daratumumab and bortezomib have a non-overlapping mechanism of action to melflufen, the ANCHOR trial (OP-104; NCT03481556) has been designed. This Phase I/II study described the safety and the efficacy of the association melflufen/dexamethasone in combinations with daratumumab or bortezomib in RRMM patients. ${ }^{64}$ Eligible patients had to be refractory to an IMiD and/or a proteasome inhibitor (only applies to patients in the daratumumab cohort) and have received 14 prior lines of therapy. Patients assigned to the daratumumab arm could not have received prior anti-CD38 mAb therapy. Melflufen was administered intravenously at the dose of 30 or $40 \mathrm{mg}$ on day 1 of each 28-day cycle. In the proteasome inhibitor cohort, bortezomib was administered subcutaneously at the dose of $1.3 \mathrm{mg} / \mathrm{m} 2$ and dexamethasone $20 \mathrm{mg}$ ( $12 \mathrm{mg}$ if aged $\geq 75$ years) on days $1,4,8$, and $11+$ dexamethasone $40 \mathrm{mg}$ ( $20 \mathrm{mg}$ if aged $\geq 75$ years) on days 15 and 22. In the daratumumab cohort, daratumumab was administered intravenously at the dose of $16 \mathrm{mg} / \mathrm{kg}$ once weekly ( 8 doses), subsequently every 2 weeks ( 8 doses), finally every 4 weeks in combination with dexamethasone at the dose of $40 \mathrm{mg}$ ( $20 \mathrm{mg}$ if aged $\geq 75$ years) weekly. Out of 43 enrolled patients, 10 melflufen $(30 \mathrm{mg}$, $\mathrm{n}=3 ; 40 \mathrm{mg}, \mathrm{n}=7$ ) in combination with bortezomib and 
dexamethasone, and 33 received melflufen $(30 \mathrm{mg}, \mathrm{n}=6$; $40 \mathrm{mg}, \mathrm{n}=27$ ) in combination with daratumumab and dexamethasone. ${ }^{64}$

The ten patients enrolled in the bortezomib cohort showed a median age of 71 years and a median number of prior lines of 2.5. Forty\% of cases had a high-risk cytogenetic; $70 \%$ were refractory to the last line of therapy, and 30\% underwent ASCT. No dose-limiting toxicities were observed. The ORR was $60 \%$, with 3 patients achieving a VGPR and 3 a PR. PFS data were not reported. The most common grade 3/4 treatment-related AEs were clinically manageable hematologic events: thrombocytopenia $(80 \%)$, neutropenia $(60 \%)$, and anemia $(40 \%)$, while grade $3 / 4$ nonhematologic treatment-related AEs were uncommon. A serious treatment-related AEs was observed in $6(60 \%)$ patients, most commonly pneumonia $(20 \%){ }^{64}$

The 33 patients enrolled in the daratumumab cohort showed a median age of 64 years and a median number of prior lines of 2 . Forty-two $\%$ of patients showed a high-risk cytogenetic; $61 \%$ were refractory to the last line of therapy, and $79 \%$ received ASCT. No doselimiting toxicities were reported. The ORR was $70 \%$, with 1 patient achieving a stringent complete response, 1 a complete response, 10 very good partial responses (VGPRs), and 11 a PR. At a median follow-up of 11.9 months, the median PFS was 11.5 months $(95 \% \mathrm{CI}$, 6.7-not reached [NR]). The median duration of response was 12.5 months $(95 \% \mathrm{CI}, 8.3-\mathrm{NR})$. Also, in this arm, the most common grade 3/4 treatment-related AEs were hematologic events: neutropenia (58\%), thrombocytopenia (55\%), and anemia (24\%), while grade $3 / 4$ nonhematologic treatment-related AEs were uncommon. A serious treatment-related AEs was observed in 20 (36\%) patients, most commonly infectious diseases. Influenza in $9 \%$ of cases, pneumonia, parainfluenza virus infection, and febrile neutropenia in $6 \%$ each. These preliminary results showed as both triplet regimens have encouraging activity in heavily pretreated RRMM and were well tolerated. ${ }^{64}$

\section{Additional Clinical Development OCEAN Trial}

OCEAN trial (OP-103; NCT03151811) is a randomized, head-to-head, superiority, open-label, global Phase III study designed to evaluate the safety and the efficacy of melflufen plus dexamethasone versus pomalidomide plus dexamethasone in RRMM patients who have received 2-4 previous lines of therapy and are refractory to both lenalidomide and their last treatment. ${ }^{44}$ The first participant was randomized into this trial study in June 2017. The study has a projected completion date of March 2022. ${ }^{65}$

However, in May 2021, a press release announced that according to the Independent Review Committee Assessment, melflufen was non-inferior to pomalidomide on the primary endpoint of PFS, with a Hazard Ratio of 0.817 in favor of melflufen. The ORR was $32.1 \%$ for melflufen versus $26.5 \%$ for pomalidomide. Moreover, the melflufen safety profile was in line with previous studies. Finally, melflufen and pomalidomide had similar discontinuation rates due to AEs (https://www.oncopeptides.com/ en/media/press-releases).

\section{BRIDGE Trial}

BRIDGE (OP-107; NCT03639610), a multicenter Phase II study, aimed to evaluate the pharmacokinetics, the safety, and the efficacy of melflufen plus dexamethasone in RRMM patients. Among the inclusion criteria, patients must have received at least two lines of treatment and have a renal impairment. ${ }^{66}$ Patients with a renal function (evaluated by creatinine clearance by Cockcroft-Gault formula) between 30 and $45 \mathrm{~mL} / \mathrm{min}$ were allocated to cohort 1 , and those between 15 and $30 \mathrm{~mL} / \mathrm{min}$ to cohort 2 . The study started in August 2018 and will include up to 25 patients. The last patient is expected to be enrolled in March 2021. Since renal impairment is a common complication of MM patients, ${ }^{67}$ the BRIDGE study will demonstrate how melflufen can be used in the setting of RRMM patients with renal insufficiency. ${ }^{66}$

\section{LIGHTHOUSE Trial}

LIGHTHOUSE (OP-108) is a multicenter, randomized, open-label, Phase 3 study comparing the efficacy and safety of melflufen and dexamethasone in combination with subcutaneous daratumumab versus subcutaneous daratumumab in RRMM patients' double refractory (or intolerant) to an IMiD and a PI or who have received $\geq 3$ prior lines of therapy including an IMiD and a PI. ${ }^{68}$ This trial has been designed to confirm the preliminary results of the ANCHOR study for the association between melflufen, daratumumab, and dexamethasone in a cohort of RRMM patients who showed similar characteristics to that of the indication for daratumumab monotherapy. ${ }^{68}$ 


\section{Safety and Tolerability}

Since RRMM patients showed a reduced bone marrow reserve due to both $\mathrm{MM}$ disease and side effects of previous lines of therapy, the hematological toxicity during melflufen treatment is most common. ${ }^{62}$ Given that cytopenias are the most common toxicities in patients treated with melflufen, monitoring for these and providing appropriate management and supportive care with growth factor support and transfusions are recommended. ${ }^{61-64}$ Although melflufen recognizes an alkylator-dependent mechanism of action, it does not seem to cause alopecia and clinically significant mucositis. ${ }^{61-64}$

\section{Regulatory Status}

In February 2021, the US FDA has approved melflufen in combination with dexamethasone for the treatment of adults with RRMM already exposed at least to four lines of therapy and refractory minimum to one proteasome inhibitor, one immunomodulatory agent, and one CD-38 directed monoclonal antibody. In April 2021, an application to the European Medicines Agency for conditional marketing authorization of melflufen in Europe has been submitted. ${ }^{38}$

\section{Conclusion}

Although both patients and hematologists settle the convenience of effective therapeutic algorithms, the heterogeneity of $\mathrm{MM}$ also imposes challenges. In this respect, despite continuous therapeutic improvements, some patients still experience shortened responses to therapy, recurrent relapses, and short overall survival. The majority of them are likely to be triple-class refractory in the course of the disease, having already exploited triplet or quadruplet combinations of effective agents.

The FDA has granted approval to melflufen in combination with dexamethasone for RRMM patients, who have received at least four prior lines of therapy, and refractory to at least one proteasome inhibitor, one immunomodulatory agent, and one anti-CD38 monoclonal antibody. ${ }^{36}$ Furthermore, combination therapy with other standard treatments, ie, bortezomib and daratumumab (ANCHOR trial), remarkably performed. ${ }^{64}$ Finally, future trials (LIGHTHOUSE trial or OCEAN trial) are ongoing to confirm, in agreement with primary analysis, clinically meaningful usefulness results as well as safety profiles of the above-mentioned therapeutic combinations. The absence of alopecia and of clinically significant mucositis, and the low infection rate are far considered trivial aspects. This notion is crucial in the present era in which novel therapies have to be applied in patients already experiencing multiple lines of treatment. Ultimately, melflufen increases the list of novel treatments, with more problematic in terms of toxicity, such as panobinostat ${ }^{69}$ and belantamab mafodotin, ${ }^{19,70}$ potentially exploitable in heavily pretreated RRMM patients.

\section{Acknowledgments}

This work was supported by: Associazione Italiana Ricerca sul Cancro (AIRC) Grant $5 \times$ mille ID.9980, (F.M., and A.N.); AIRC I.G. ID.16722 and IG24365 (to A.N.); AIRC and Fondazione CaRiCal co-financed Multi-Unit Regional Grant 2014 n.16695 (to F.M.); Italian Ministry of Health $5 \times 1000$ funds 2014 and funds 2016 (to G.C.); Italian Ministry of Health Alleanza Contro il Cancro (ACC), hematology network; Italian Ministry of Health, Current Research (to G.C and A.N.).

\section{Disclosure}

The authors report no conflicts of interest in this work.

\section{References}

1. Moreau P, Zamagni E, Mateos MV. Treatment of patients with multiple myeloma progressing on frontline-therapy with lenalidomide. Blood Cancer J. 2019;9(4):38. doi:10.1038/s41408-019-0200-1

2. Gentile M, Vigna E, Recchia AG, Morabito L, Martino M, Morabito F. Role of new drugs incorporated into consolidation and maintenance therapy in transplant-eligible multiple myeloma patients. Expert Opin Pharmacother. 2014;15(10):1315-1320. doi:10.1517/14656566.2014. 919257

3. Gentile M, Morabito F, Martino M, et al. Chemotherapy-based regimens in multiple myeloma in 2020. Panminerva Med. 2021;63 (1):7-12. doi:10.23736/S0031-0808.20.04145-2

4. Gentile M, Offidani M, Vigna E. Ixazomib for the treatment of multiple myeloma. Expert Opin Investig Drugs. 2015;24(9):1287-1298. doi:10.1517/13543784.2015.1065250

5. Gentile M, Specchia G, Derudas D, et al. Elotuzumab, lenalidomide, and dexamethasone as salvage therapy for patients with multiple myeloma: Italian, multicenter, retrospective clinical experience with 300 cases outside of controlled clinical trials. Haematologica. 2021;106(1):291-294. doi:10.3324/haematol.2019.241513

6. Botta C, Martino EA, Conticello C, et al. Treatment of lenalidomide exposed or refractory multiple myeloma: network meta-analysis of lenalidomide-sparing regimens. Front Oncol. 2021;11:643490. doi: 10.3389 /fonc. 2021.643490

7. Markovic U, Romano A, Del Fabro V, et al. Daratumumab as single agent in relapsed/refractory myeloma patients: a retrospective real-life survey. Front Oncol. 2021;11:624405. doi:10.3389/fonc.2021.624405

8. Palmieri S, Rocco S, Vitagliano O, et al. KRD (carfilzomib and lenalidomide plus dexamethasone) for the treatment of relapsed or refractory multiple myeloma in the real-life: a retrospective survey in 123 patients. Ann Hematol. 2020;99(12):2903-2909. doi:10.1007/ s00277-020-04158-4

9. Bringhen S, D'Agostino M, Paris L, et al. Lenalidomide-based induction and maintenance in elderly newly diagnosed multiple myeloma patients: updated results of the EMN01 randomized trial. Haematologica. 2020;105(7):1937-1947. doi:10.3324/haematol.2019.226407 
10. Gentile M, Magarotto V, Offidani M, et al. Lenalidomide and low-dose dexamethasone ( $\mathrm{Rd}$ ) versus bortezomib, melphalan, prednisone (VMP) in elderly newly diagnosed multiple myeloma patients: a comparison of two prospective trials. Am J Hematol. 2017;92 (3):244-250. doi:10.1002/ajh.24621

11. Uccello G, Petrungaro A, Mazzone C, et al. Pomalidomide in multiple myeloma. Expert Opin Pharmacother. 2017;18(2):133-137. doi:10.1080/14656566.2016.1274973

12. Pozzi S, Gentile M, Sacchi S, et al. Bendamustine, Low-dose dexamethasone, and lenalidomide (BdL) for the treatment of patients with relapsed/refractory multiple myeloma confirms very promising results in a phase I/II study. Leuk Lymphoma. 2017;58(3):552-559. doi:10.1080/10428194.2016.1205741

13. Gentile M, Martino M, Recchia AG, Vigna E, Morabito L, Morabito F. Sorafenib for the treatment of multiple myeloma. Expert Opin Investig Drugs. 2016;6:1-7.

14. Gentile M, Recchia AG, Mazzone C, et al. An old drug with a new future: bendamustine in multiple myeloma. Expert Opin Pharmacother. 2013;14(16):2263-2280. doi:10.1517/14656566.2013.837885

15. Gentile M, Vigna E, Recchia AG, et al. Bendamustine in multiple myeloma. Eur J Haematol. 2015;95(5):377-388. doi:10.1111/ejh. 12609

16. Morabito F, Bringhen S, Larocca A, et al. Bortezomib, melphalan, prednisone (VMP) versus melphalan, prednisone, thalidomide (MPT) in elderly newly diagnosed multiple myeloma patients: a retrospective case-matched study. Am J Hematol. 2014;89(4): 355-362. doi:10.1002/ajh.23641

17. Jasiński M, Basak GW, Jedrzejczak WW. Perspectives for the Use of CAR-T cells for the treatment of multiple myeloma. Front Immunol. 2021;12:632937. doi:10.3389/fimmu.2021.632937

18. Maples KT, Johnson C, Lonial S. Antibody treatment in multiple myeloma. Clin Adv Hematol Oncol. 2021;19(3):166-174.

19. Lassiter G, Bergeron C, Guedry R, et al. Belantamab mafodotin to treat multiple myeloma: a comprehensive review of disease, drug efficacy and side effects. Curr Oncol. 2021;28(1):640-660. doi:10. 3390/curroncol28010063

20. Devarakonda S, Efebera Y, Sharma N. Role of stem cell transplantation in multiple myeloma. Cancers (Basel). 2021;13(4):863. doi: $10.3390 /$ cancers 13040863

21. Martino M, Tripepi G, Messina G, et al. A Phase II, single-arm, prospective study of bendamustine plus melphalan conditioning for second autologous stem cell transplantation in de novo multiple myeloma patients through a tandem transplant strategy. Bone Marrow Transplant. 2016;51(9):1197-1203. doi:10.1038/bmt.2016.94

22. Martino M, Recchia AG, Fedele R, et al. The role of tandem stem cell transplantation for multiple myeloma patients. Expert Opin Biol Ther. 2016;16(4):515-534. doi:10.1517/14712598.2016.1136285

23. Martino M, Olivieri A, Offidani M, et al. Addressing the questions of tomorrow: melphalan and new combinations as conditioning regimens before autologous hematopoietic progenitor cell transplantation in multiple myeloma. Expert Opin Investig Drugs. 2013;22 (5):619-634. doi:10.1517/13543784.2013.788643

24. Martino M, Recchia AG, Console G, et al. Can we improve the conditioning regimen before autologous stem cell transplantation in multiple myeloma? Expert Opin Orph Drugs. 2017;5(11):875-887. doi:10.1080/21678707.2017.1387050

25. Furner RL, Brown RK. L-phenylalanine mustard (L-PAM): the first 25 years. Cancer Treat Rep. 1980;64(4-5):559-574.

26. Musto P, D'Auria F. Melphalan: old and new uses of a still master drug for multiple myeloma. Expert Opin Investig Drugs. 2007;16 (9):1467-1487. doi:10.1517/13543784.16.9.1467

27. Martino M, Postorino M, Gallo GA, et al. Long-term results in multiple myeloma after high-dose melphalan and autologous transplantation according to response categories in the era of old drugs. Clin Lymphoma Myeloma Leuk. 2014;14(2):148-154. doi:10.1016/j. clm1.2013.11.009
28. Sporn JR, McLntyre OR. Chemotherapy of previously untreated multiple myeloma patients: an analysis of recent treatment results. Semin Oncol. 1986;13(3):318-325.

29. Mateos MV, Dimopoulos MA, Cavo M, et al. Daratumumab plus bortezomib, melphalan, and prednisone for untreated myeloma. N Engl J Med. 2018;378(6):518-528. doi:10.1056/NEJMoa1714678

30. Palumbo A, Bringhen S, Rossi D, et al. Bortezomib-melphalanprednisone-thalidomide followed by maintenance with bortezomib-thalidomide compared with bortezomib-melphalanprednisone for initial treatment of multiple myeloma: a randomized controlled trial. J Clin Oncol. 2010;28(34):5101-5109. doi:10.1200/ JCO.2010.29.8216

31. McElwain TJ, Powles RL. High-dose intravenous melphalan for plasma-cell leukaemia and myeloma. Lancet. 1983;2 (8354):822-824. doi:10.1016/S0140-6736(83)90739-0

32. Kazandjian D, Mo CC, Landgren O, Richardson PG. The role of high-dose melphalan with autologous stem-cell transplant in multiple myeloma: is it time for a paradigm shift? Br J Haematol. 2020;191 (5):692-703. doi:10.1111/bjh.16764

33. Gullbo J, Wickström M, Tullberg M, et al. Activity of hydrolytic enzymes in tumour cells is a determinant for anti-tumour efficacy of the melphalan containing prodrug J1. J Drug Target. 2003;11 (6):355-363. doi:10.1080/10611860310001647140

34. Gullbo J, Dhar S, Luthman K, et al. Antitumor activity of the alkylating oligopeptides J1 (L-melphalanyl-p-L-fluorophenylalanine ethyl ester) and P2 (L-prolyl-m-L-sarcolysyl-p-L-fluorophenylalanine ethyl ester): comparison with melphalan. Anticancer Drugs. 2003;14 (8):617-624. doi:10.1097/00001813-200309000-00006

35. Gullbo J, Lindhagen E, Bashir-Hassan S, et al. Antitumor efficacy and acute toxicity of the novel dipeptide melphalanyl-p-L-fluorophenylalanine ethyl ester (J1) in vivo. Invest New Drugs. 2004;22(4):411-420. doi:10.1023/B:DRUG.0000036683.10945.bb

36. Wickstrom M, Viktorsson K, Lundholm L, et al. The alkylating prodrug $\mathrm{J} 1$ can be activated by aminopeptidase $\mathrm{N}$, leading to a possible target directed release of melphalan. Biochem Pharmacol. 2010;79(9):1281-1290. doi:10.1016/j.bcp.2009.12.022

37. Wickstrom M, Nygren P, Larsson R, et al. Melflufen-A peptidasepotentiated alkylating agent in clinical trials. Oncotarget. 2017;8 (39):66641-66655. doi:10.18632/oncotarget.18420

38. FDA approves Oncopeptides' PEPAXTO $^{\circledR}$ (melphalan flufenamide) for patients with relapsed or refractory multiple myeloma. News release. Oncopeptides, AB; February 26, 2021. Accessed February 26, 2021.

39. De Barbieri A Peptichemio: a synthesis of pharmacological, morphological, biochemical and biomolecular investigations. In: Proceedings from the Symposium on Peptichemio, Milan, Italy, 1972.

40. Hansson J, Lewensohn R, Ringborg U. Cytotoxicity and DNA cross-linking induced by peptide conjugated $\mathrm{m}$-L-sarcolysin in human melanoma cells. Anticancer Res. 1991;11(5):1725-1730.

41. Lewensohn R, Ehrsson H, Hansson J, Ringborg U. Increased toxicity and DNA cross-linking by peptide bound m-L-sarcolysin (Peptichemio) as compared to melphalan and m-L-sarcolysin in human melanoma cell lines. Anticancer Res. 1991;11(1):321-324.

42. Wickström M, Haglund C, Lindman H, Nygren P, Larsson R, Gullbo J. The novel alkylating prodrug $\mathrm{J} 1$ : diagnosis directed activity profile ex vivo and combination analyses in vitro. Invest New Drugs. 2008;26(3):195-204. doi:10.1007/s10637-007-9092-1

43. Wickstrom M, Larsson R, Nygren P, Gullbo J. Aminopeptidase $\mathrm{N}$ (CD13) as a target for cancer chemotherapy. Cancer Sci. 2011;102(3):501-508. doi:10.1111/j.1349-7006.2010.01826.x

44. Mina-Osorio P. The moonlighting enzyme CD13: old and new functions to target. Trends Mol Med. 2008;14(8):361-371. doi:10.1016/j. molmed.2008.06.003

45. Chauhan D, Ray A, Viktorsson K, et al. In vitro and in vivo antitumor activity of a novel alkylating agent, melphalan-flufenamide, against multiple myeloma cells. Clin Cancer Res. 2013;19(11):3019-3031. doi:10.1158/1078-0432.CCR-12-3752 
46. Miettinen JJ, Kumari R, Traustadottir GA, et al. Aminopeptidase expression in multiple myeloma associates with disease progression and sensitivity to melflufen. Cancers (Basel). 2021;13(7):1527. doi:10.3390/cancers 13071527

47. Kumari R, Majumder MM, Lievonen J, et al. Prognostic significance of esterase gene expression in multiple myeloma. $\mathrm{Br} J$ Cancer. 2021;124(8):1428-1436. doi:10.1038/s41416-020-01237-1

48. Gullbo J, Tullberg M, Vabeno J, et al. Structure-activity relationship for alkylating dipeptide nitrogen mustard derivatives. Oncol Res. 2003;14(3):113-132. doi:10.3727/000000003771013071

49. Moore HE, Davenport EL, Smith EM, et al. Aminopeptidase inhibition as a targeted treatment strategy in myeloma. Mol Cancer Ther. 2009;8(4):762-770. doi:10.1158/1535-7163.MCT-08-0735

50. Berglund Å, Ullén A, Lisyanskaya A, et al. First-in-human, phase I/ IIa clinical study of the peptidase potentiated alkylator melflufen administered every three weeks to patients with advanced solid tumor malignancies. Invest New Drugs. 2015;33(6):1232-1241. doi:10.1007/s10637-015-0299-2

51. Gullbo J. Preclinical development of new alkylating oligopeptides for cancer therapy. Medical Sciences, Dept of Clinical PharmacologyUppsala: Uppsala University; 2003.

52. Wickstrom M, Johnsen JI, Ponthan F, et al. The novel melphalan prodrug J1 inhibits neuroblastoma growth in vitro and in vivo. Mol Cancer Ther. 2007;6(9):2409-2417. doi:10.1158/1535-7163.MCT$07-0156$

53. Byrgazov K, Anderson C, Salzer B, et al. Targeting aggressive osteosarcoma with a peptidase-enhanced cytotoxic melphalan flufenamide. Ther Adv Med Oncol. 2020;12:1758835920937891. doi:10.1177/1758835920937891

54. Wang S, Xie H, Xing Wei X, et al. Relation between the expression of aminopeptidase $\mathrm{N}$ (APN)/CD13 and the clinical significance in osteosarcomas. Int J Clin Exp Med. 2016;9:22034-22040.

55. Falco P, Bringhen S, Avonto I, et al. Melphalan and its role in the management of patients with multiple myeloma. Expert Rev Anticancer Ther. 2007;7(7):945-957. doi:10.1586/14737140.7.7.945

56. Hazlehurst LA, Enkemann SA, Beam CA, et al. Genotypic and phenotypic comparisons of de novo and acquired melphalan resistance in an isogenic multiple myeloma cell line model. Cancer Res. 2003;63(22):7900-7906.

57. Jones RB. Clinical pharmacology of melphalan and its implications for clinical resistance to anticancer agents. Cancer Treat Res. 2002;112:305-322.

58. McHugh PJ, Spanswick VJ, Hartley JA. Repair of DNA interstrand crosslinks: molecular mechanisms and clinical relevance. Lancet Oncol. 2001;2(8):483-490. doi:10.1016/S1470-2045(01)00454-5

59. Spanswick VJ, Craddock C, Sekhar M, et al. Repair of DNA interstrand crosslinks as a mechanism of clinical resistance to melphalan in multiple myeloma. Blood. 2002;100(1):224-229. doi:10.1182/ blood.V100.1.224
60. Ray A, Das DS, Song Y, et al. A novel alkylating agent Melflufen induces irreversible DNA damage and cytotoxicity in multiple myeloma cells. $\mathrm{Br} J$ Haematol. 2016;174(3):397-409. doi:10.1111/ bjh. 14065

61. Richardson PG, Bringhen S, Voorhees P, et al. Melflufen plus dexamethasone in relapsed and refractory multiple myeloma (O-12-M1): a multicentre, international, open-label, phase 1-2 study. Lancet Haematol. 2020;7(5):e395-e407. doi:10.1016/S2352-3026(20)300 44-2

62. Bringhen S, Voorhees PM, Plesner T, et al. Melflufen plus dexamethasone in relapsed/ refractory multiple myeloma: long-term survival follow-up from the Phase II study O-12-M1. $\mathrm{Br} J$ Haematol. 2021;193(6):1105-1109. doi:10.1111/bjh.17302

63. Richardson PG, Oriol A, Larocca A, et al. Melflufen and dexamethasone in heavily pretreated relapsed and refractory multiple myeloma. J Clin Oncol. 2021;39(7):757-767. doi:10.1200/JCO.20.02259

64. Ocio EM, Efebera YA, Hajek R, et al. ANCHOR (OP-104): melflufen plus dexamethasone (dex) and Daratumumab (dara) or Bortezomib (BTZ) in Relapsed/ Refractory Multiple Myeloma (RRMM) Refractory to an IMiD and/or a Proteasome Inhibitor (PI) - Updated Efficacy and Safety. Blood. 2020;136(suppl 2):abs 417. doi:10.1182/ blood-2020-135991

65. Schjesvold F, Robak P, Pour L, Aschan J, Sonneveld P. OCEAN: a randomized Phase III study of melflufen + dexamethasone to treat relapsed refractory multiple myeloma. Future Oncol. 2020;16 (11):631-641. doi:10.2217/fon-2020-0024

66. NIH Clinical Trials. A PK study of melphalan during treatment with Melphalan Flufenamide (Melflufen) and Dex in RRMM pat with impaired renal function. https://clinicaltrials.gov/ct $2 / \mathrm{show} /$ NCT03639610. Accessed June 25, 2021.

67. Dimopoulos MA, Sonneveld P, Leung N, et al. International myeloma working group recommendations for the diagnosis and management of myeloma-related renal impairment. J Clin Oncol. 2016;34 (13):1544-1557. doi:10.1200/JCO.2015.65.0044

68. Mateos MV, Ocio EM, Sonneveld P, et al. LIGHTHOUSE (OP-108): a phase 3 study of melflufen in combination with daratumumab versus daratumumab in patients with relapsed/refractory multiple myeloma. EHA Congress; 2020; abs PB2018.

69. San-Miguel JF, Hungria VT, Yoon SS, et al. Panobinostat plus bortezomib and dexamethasone versus placebo plus bortezomib and dexamethasone in patients with relapsed or relapsed and refractory multiple myeloma: a multicentre, randomised, double-blind phase 3 trial. Lancet Oncol. 2014;15(11):1195-1206. doi:10.1016/S14702045(14)70440-1

70. Lonial S, Lee HC, Badros A, et al. Belantamab mafodotin for relapsed or refractory multiple myeloma (DREAMM-2): a two-arm, randomised, open-label, phase 2 study. Lancet Oncol. 2020;21 (2):207-221. doi:10.1016/S1470-2045(19)30788-0

\section{Publish your work in this journal}

Drug Design, Development and Therapy is an international, peerreviewed open-access journal that spans the spectrum of drug design and development through to clinical applications. Clinical outcomes, patient safety, and programs for the development and effective, safe, and sustained use of medicines are a feature of the journal, which has also been accepted for indexing on PubMed Central. The manuscript management system is completely online and includes a very quick and fair peer-review system, which is all easy to use. Visit http://www. dovepress.com/testimonials.php to read real quotes from published authors. 